\title{
FORCE CONTROL AND EXPONENTIAL STABILITY FOR ONE-LINK FLEXIBLE ARM
}

\author{
Takahiro Endo* Fumitoshi Matsuno **
}

\author{
* Tokyo Institute of Technology, \\ Nagatsuta, Midori, Yokohama, 226-8502, JAPAN \\ ** The University of Electro-Communications, \\ Chofugaoka, Chofu, Tokyo, 182-8585, JAPAN
}

\begin{abstract}
We discuss a force control problem for a constrained one-link flexible arm based on distributed parameter model. In order to solve the force control problem, we propose a simple feedback control law. This control law consists of bending moment at the root of the flexible arm and its derivative, which regulates simultaneously the force and the rotational angle of the motor without the angle and the force information. Then it provides the exponential stability of the closed-loop system by using energy multiplier method. As the control law is derived based on the distributed parameter model, we can avoid the drawbacks resulting from finite dimensional approximation. Copyright (c)2005 IFAC
\end{abstract}

Keywords: Flexible arms, Force control, Exponentially stable, Distributed parameter systems, Partial differential equations

\section{INTRODUCTION}

It is necessary for industrial and space manipulators to be manufactured from lightweight material because of demands for low-energy consumption and limitation on carrying capacity of space rockets. An elasticity of such elements gives rise to problem of an undesired vibration. Dynamics of arms with distributed elasticity are expressed as distributed parameter systems. However the controller of such distributed parameter systems is generally designed based on finite dimensional approximated model. In this case how infinite dimensional systems is approximated to finite dimensional systems is one of important topics.

Finite dimensional approximated model is derived by neglecting infinite modes. Therefore the following problems arise: the dimension of the controller increases as the number of the modes, which are considered in the controller design model, increases, spillover occurred by the disregarded high frequency characteristic makes a system unstable and so on (Balas, 1978). From this point of view the controller design based on infinite dimensional systems is desired. For vibration or vibration and tip position control of flexible arm, various controllers have been proposed (Morgül, 1991; Luo, 1993; Luo and Guo, 1997; Luo and Feng, 1999) and asymptotic/exponential stability is shown.

On the other hand only vibration and tip position control are not enough to use flexible arms in more complex task. It is also necessary to control the contact force which an end-effector of the flexible arms exerts on an object or environment. For force control of a constrained one-link flexible arm, although there are some researches based on finite dimensional approximated model (Chiou and Shahinpoor, 1988; Matsuno and Kasai, 1998; Morita et al., 2001; Siciliano and Villani, 2000), there are few researches based on distributed parameter model (Morita et al., 2002). In Morita et al. (2002) the force control problem for one-link flexible arm is discussed based on distributed parameter model. And the asymptotic stabilizing controller of the closed-loop system is constructed using Lyapunov method. However it is better to construct the 
controller which exponentially stabilize the closedloop system, in the sense of control performance. So we discuss the force control problem for the flexible arm based on distributed parameter model and show that the exponential stability of the closed-loop system can be accomplished by the simple feedback control law.

In this paper we consider the design of control law and exponential stability of the closed-loop system for the constrained one-link flexible arm. We design the controller based on an original distributed parameter model, we can avoid the above drawbacks. The paper is organized as follows. In Section 2 we describe the mathematical model of the constrained one-link flexible arm. Since we consider the force control problem of the arm, our aim is to design controller which regulates the constraint force to a desired one, and achieves the vibration absorption when the arm is rotated in the horizontal plane. Then in order to accomplish this control objective we propose a simple feedback control law and prove the exponential stability of the closed-loop system by using energy multiplier method in Section 3. Finally Section 4 presents conclusions.

\section{DESCRIPTION OF PROBLEM}

\subsection{Dynamics of Constrained Flexible Arm}

Constrained one-link flexible arm which we consider in this paper has been illustrated in Fig.1. One end of this flexible arm is clamped to the control motor and the other end has a concentrated mass $m$. The tip mass is making contact with a surface of the object. Using this control motor the arm rotates in the horizontal plane and are not affected by the acceleration of gravity. The flexible arm, having length $l$, uniform linear mass density $\rho$, and uniform flexural rigidity $E I$, satisfies the Euler-Bernoulli beam hypothesis which ignores the moment of inertia and shear deformation of the arm.

Denote by $O-X Y$ a world coordinate system, and by $O-x y$ a local coordinate system as shown in Fig.1. The origin of $O-x y$ is fixed at the rotor of the control motor and $O$ - $x y$ rotates with the rotor. Let $J, \tau_{a}(t), \theta(t)$ and $w(x, t)$ be the moment of inertia of the rotor of the motor, the torque caused by the motor, the rotational angle of the motor and the transverse displacement of

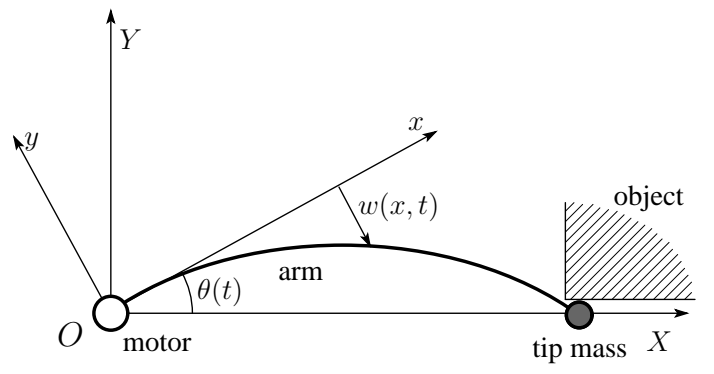

Fig. 1. Constrained one-link flexible arm. the arm at time $t$ and at a spatial point $x$, respectively. Note that $\theta(t)$ and $w(x, t)$ are assumed to be small.

Since the tip mass is making contact with the surface, we obtain a geometric constraint as shown in the following:

$$
\phi \equiv l \theta(t)-w(l, t)=0 .
$$

Under the above preparation we can obtain the equations of motion (Matsuno and Kasai, 1998):

$$
\begin{aligned}
& w_{t t}(x, t)+\frac{E I}{\rho} w_{x x x x}(x, t)=x \ddot{\theta}(t), \\
& w(0, t)=w_{x}(0, t)=w_{x x}(l, t)=0, \\
& m\left\{l \ddot{\theta}(t)-w_{t t}(l, t)\right\}+E I w_{x x x}(l, t)=\lambda(t), \\
& J \ddot{\theta}(t)+\mu \dot{\theta}(t)=\tau(t),
\end{aligned}
$$

where $\mu$ is the viscous friction coefficient, $\tau(t)=$ $\tau_{a}(t)-E I w_{x x}(0, t)$, a dot denotes the time derivative and the subscript denotes the partial derivative with respect to the corresponding variable. Equation (2) describes the bending vibration of the arm and (3), (4) represent the boundary conditions. Equation (5) gives a description of the equation of rotation of the motor. In equation (4) $\lambda(t)$ is the Lagrange multiplier associated with the constraint $\phi$. From equation (1) and (4) it is found that $\lambda(t)=E I w_{x x x}(l, t), \lambda(t)$ is equivalent to the contact force which arise in the direction in the normal vector to the constraint surface. Substituting this relation into (4) gives

$$
l \ddot{\theta}(t)-w_{t t}(l, t)=0 .
$$

This equation is equivalent to the two time derivative of constraint $\phi$, and hereinafter we use this constraint instead of (4). By introducing the new variable

$$
y(x, t)=w(x, t)-x \theta(t),
$$

the equations of motion can be rewritten as

$$
\begin{aligned}
& y_{t t}(x, t)+\frac{E I}{\rho} y_{x x x x}(x, t)=0, \\
& y(0, t)=y(l, t)=y_{x x}(l, t)=0, \\
& J y_{x t t}(0, t)+\mu y_{x t}(0, t)=-\tau(t) .
\end{aligned}
$$

\subsection{Control Objective}

The aim of this paper is to control the contact force. In other words the control objective is to derive the control law such that the $\lambda(t), w_{t}(x, t)$ and $\dot{\theta}(t)$ satisfies the following relations:

$$
\lambda(t) \rightarrow \lambda^{d}, w_{t}(x, t) \rightarrow 0, \dot{\theta}(t) \rightarrow 0,
$$

where $\lambda^{d}$ is the constant desired contact force. Since the equation $y_{t}(x, t)=0$ is hold in the desired states from (10), $y(x, t)$ becomes function of only variable $x$ and describes it as $y^{s}(x)$. By substituting (10) into the equations of motion (7), (8) and considering $\lambda(t)=$ $E I w_{x x x}(l, t)=E I y_{x x x}(l, t)$, we obtain the equation

$$
y^{s}(x)=\frac{\lambda^{d}}{6 E I} x\left(2 l^{2}-3 l x+x^{2}\right) .
$$


This is a static transverse displacement of $y(x, t)$ in the case that the force converges to the desired value $\left(\lambda(t) \rightarrow \lambda^{d}\right)$. Let $y^{d}(x)$ be $y^{s}(x)$. Then control objective (10) is accomplished by constructing the control law such as $y(x, t) \rightarrow y^{d}(x)$ and $y_{t}(x, t) \rightarrow 0$. It is one character of the force control of flexible arms that the static transverse displacement is treated as objective variable for controlling the contact force, and this is a different character from the case of rigid robot arms.

On the other hand when flexible arm generates desired contact force $\lambda^{d}$, the static rotational angle of the arm is as follows:

$$
\theta^{d}=-y_{x}^{d}(0)=-\frac{l^{2} \lambda^{d}}{3 E I} .
$$

From (11) and (12) we found that desired static transverse displacement and desired rotational angle are coupling through $\lambda^{d}$. Thus it turns out that rotational angle and contact force are uncontrollable to arbitrary point if we want to set the contact force and rotational angle to desired one, independently.

\section{FORCE CONTROL}

\subsection{Proposed Control Law}

We construct the control law which satisfies following relations:

$$
y(x, t) \rightarrow y^{d}(x), y_{t}(x, t) \rightarrow 0 .
$$

If $y_{t}(x, t)=0$ is satisfied then $y(x, t)=y^{s}(x)$ and it is easy to see that $y^{s}(x)=y^{d}(x)$, provided that $y_{x x}^{s}(0)=y_{x x}^{d}(0)$. Therefore we can rewrite the relations (13) as

$$
y_{x x}(0, t) \rightarrow y_{x x}^{d}(0), y_{t}(x, t) \rightarrow 0 .
$$

We propose the following feedback control law to accomplish the relations (14):

$$
\begin{aligned}
\tau(t)=-k_{1} E I\left\{y_{x x}(0, t)\right. & \left.-y_{x x}^{d}(0)\right\} \\
& -k_{2} E I y_{x x t}(0, t),
\end{aligned}
$$

where feedback gain $k_{1}$ and $k_{2}$ are positive constants. In the control law (15) the first term is expected to achieve that the bending moment at the root of the arm $y_{x x}(0, t)$ converges to the desired $y_{x x}^{d}(0)$. The second term is also the term for the absorption of the bending vibration $\left(y_{t}(x, t) \rightarrow 0\right)$ and it is known that this term is effective for vibration absorption in the case of one-link flexible arm with free end (Luo, 1993). This control law consists of bending moment at the root of the flexible arm and its derivative. The proposed control law is henceforth described as Moment-PD control because of simplicity.

It is necessary to investigate that the equilibrium point of the system under the control law (15) is desired states. Since $y_{t}, y_{t t}, y_{x t}(0, t)$ and $y_{x t t}(0, t)$ are equal to zero at equilibrium states, $y$ become function of variable $x$. Substituting them into closed-loop system then it is easy to see that the equilibrium states becomes desired states by simple calculations.

In the next subsection we will investigate about the stability of the closed-loop system. For simplicity the equilibrium point is moved to origin using

$$
y(x, t)=y(x, t)-y^{d}(x) .
$$

\subsection{Closed-Loop System}

We formulate the following closed-loop system to a first order evolution equation in an appropriate Hilbert space:

$$
\begin{aligned}
& y_{t t}(x, t)+\frac{E I}{\rho} y_{x x x x}(x, t)=0, \\
& \begin{array}{r}
y(0, t)=y(l, t)=y_{x x}(l, t)=0, \\
y_{x t t}(0, t)+\tilde{\mu} y_{x t}(0, t)-\tilde{k}_{1} E I y_{x x}(0, t) \\
\quad-\tilde{k}_{2} E I y_{x x t}(0, t)=0,
\end{array}
\end{aligned}
$$

where $\{\tilde{\cdot}\}=\{\cdot\} / J$. First we define the function

$$
\zeta(t)=-E I y_{x x}(0, t)+\frac{1}{\tilde{k}_{2}} y_{x t}(0, t),
$$

as Conrad and Morgül (1998). Substituting (20) into (19) gives

$$
\tilde{k}_{2} \dot{\zeta}(t)+\tilde{k}_{1} \zeta(t)+\left(\tilde{\mu}-\frac{\tilde{k}_{1}}{\tilde{k}_{2}}\right) y_{x t}(0, t)=0 .
$$

Next we introduce following functional space $H$ as the state space of the closed-loop system (17), (18), (20) and (21):

$$
H=\left(H^{2}(0, l) \cap H_{0}^{1}(0, l)\right) \times L^{2}(0, l) \times R .
$$

The space $H^{m}(\Omega), \Omega$ is a bounded open set in $R$, is the Sobolev space of order $m$, the space $H_{0}^{m}(\Omega)$ is the closure in $H^{m}(\Omega)$ of $C_{0}^{\infty}(\Omega)$ and the space $L^{2}(\Omega)$ is the usual square integrable functional space. In the state space $H$ we define the inner-product:

$$
\langle z, \hat{z}\rangle_{H}=\frac{E I}{2} \int_{0}^{l} u_{x x} \hat{u}_{x x} d x+\frac{\rho}{2} \int_{0}^{l} v \hat{v} d x+\frac{C}{2} \zeta \hat{\zeta},
$$

where $z=(u, v, \zeta)^{T} \in H, \hat{z}=(\hat{u}, \hat{v}, \hat{\zeta})^{T} \in H, C=$ $\tilde{k}_{2}^{2} /\left(\tilde{k}_{1}+\tilde{\mu} \tilde{k}_{2}\right)$. It can be shown that $H$ together with the inner-product given by (23) becomes a Hilbert space. Finally we define the unbounded linear operator $A: D(A) \subset H \rightarrow H$ as follows:

$$
A z=\left[\begin{array}{c}
v \\
-\frac{E I}{\rho} u_{x x x x} \\
-\frac{\tilde{k}_{1}}{\tilde{k}_{2}} \zeta-\frac{1}{\tilde{k}_{2}}\left(\tilde{\mu}-\frac{\tilde{k}_{1}}{\tilde{k}_{2}}\right) v_{x}(0)
\end{array}\right]
$$

where the domain of the operator $A$ is defined as

$$
\begin{aligned}
& D(A)=\left\{z: u \in W_{1}, v \in W_{2}, \zeta \in R ;\right. \\
& \left.\quad u_{x x}(l)=0, \zeta=-E I u_{x x}(0)+v_{x}(0) / \tilde{k}_{2}\right\}, \\
& W_{1}=H^{4}(0, l) \cap H_{0}^{1}(0, l), \\
& W_{2}=H^{2}(0, l) \cap H_{0}^{1}(0, l) .
\end{aligned}
$$


With the previous notations the closed-loop system (17), (18), (20) and (21) under the Hilbert space $H$ can be written as the first order evolution equation:

$$
\dot{z}=A z,
$$

where $z=\left(y, y_{t}, \zeta\right)^{T}$.

We have the following lemma for the properties of the closed-loop system.

Lemma 1. The operator $A$ generates a $C_{0}$-semigroup of contractions.

PROOF. According to the Lumer-Phillips theorem (Pazy, 1983) we have to show that the operator $A$ is dissipative and $R(I-A)$, the range of the operator $I-A$, is the whole space $H$. For any $z=(u, v, \zeta)^{T} \in$ $D(A)$ it follows that

$$
\begin{aligned}
\langle A z, z\rangle_{H}= & \frac{E I}{2} \int_{0}^{l} v_{x x} u_{x x} d x-\frac{E I}{2} \int_{0}^{l} u_{x x x x} v d x \\
& -\frac{C}{2}\left\{\frac{\tilde{k}_{1}}{\tilde{k}_{2}} \zeta+\frac{1}{\tilde{k}_{2}}\left(\tilde{\mu}-\frac{\tilde{k}_{1}}{\tilde{k}_{2}}\right) v_{x}(0)\right\} \zeta \\
= & -\frac{C}{2}\left\{\frac{\tilde{k}_{1}}{\tilde{k}_{2}}(E I)^{2} u_{x x}^{2}(0)+\frac{\tilde{\mu}}{\tilde{k}_{2}^{2}} v_{x}^{2}(0)\right\} \leq 0 .
\end{aligned}
$$

From this calculations we found that the operator $A$ is dissipative. Next we prove $R(I-A)=H$. For any given $\tilde{z}=(\tilde{u}, \tilde{v}, \tilde{\zeta})^{T} \in H$ we have to find $z=(u, v, \zeta)^{T} \in D(A)$ so that $(I-A) z=\tilde{z}$. Eliminating $v$ and $\zeta$ in this equation, we obtain the following equations:

$$
\begin{aligned}
& u+\frac{E I}{\rho} u_{x x x x}=\alpha, \\
& u(0)=u(l)=u_{x x}(l)=0, \\
& -E I u_{x x}(0)+\frac{1+\tilde{\mu}}{\tilde{k}_{1}+\tilde{k}_{2}} u_{x}(0)=\beta, \\
& \alpha=\tilde{u}+\tilde{v}, \beta=\frac{1}{\tilde{k}_{1}+\tilde{k}_{2}}\left\{\tilde{k}_{2} \tilde{\zeta}+(1+\tilde{\mu}) \tilde{u}_{x}(0)\right\} .
\end{aligned}
$$

Now we introduce new variable

$$
u(x)=q(l-x), \quad r=l-x,
$$

for solve the above equations. Then we get

$$
\begin{aligned}
& q^{\prime \prime \prime \prime}(r)+\frac{\rho}{E I} q(r)=\frac{\rho}{E I} \tilde{\alpha}, \\
& q(0)=q^{\prime \prime}(0)=0, \\
& q(l)=0, \\
& -E I q^{\prime \prime}(l)-k q^{\prime}(l)=\tilde{\beta},
\end{aligned}
$$

where a dash denotes the derivative with respect to the variable $r, \tilde{\alpha}$ and $\tilde{\beta}$ are translated functions related to $\alpha$ and $\beta$ by introducing new variable respectively, and $k=(1+\tilde{\mu}) /\left(\tilde{k}_{1}+\tilde{k}_{2}\right)$. The solution of (27) together with the boundary conditions (28) is given by

$$
\begin{aligned}
q(r)= & c_{1} \sin a r \cosh a r+c_{2} \cos a r \sinh a r \\
& +a \int_{0}^{r}[\sin a(r-\tau) \cosh a(r-\tau) \\
& \quad-\cos a(r-\tau) \sinh a(r-\tau)] \tilde{\alpha}(\tau) d \tau
\end{aligned}
$$

where $c^{4}=\rho / E I, a=c / \sqrt{2}$, and the parameter $c_{1}$ and $c_{2}$ are constants which are determined by the remaining boundary conditions. Substituting this solution into the boundary condition (29) and (30), we get the following matrix form relation:

$$
M\left[c_{1}, c_{2}\right]^{T}=\left[f_{1}, f_{2}\right]^{T} .
$$

It is easy to see that

$$
\begin{aligned}
\operatorname{det} M= & a^{2} E I(\cosh 2 a l-\cos 2 a l) \\
& +\frac{1}{2} a k(\sinh 2 a l-\sin 2 a l)>0,
\end{aligned}
$$

then the parameter $c_{1}$ and $c_{2}$ can be uniquely determined from

$$
\left[c_{1}, c_{2}\right]^{T}=M^{-1}\left[f_{1}, f_{2}\right]^{T} .
$$

Hence we can obtain $u(x)=q(r)$. The remaining unknows $v$ and $\zeta$ can be found by using $u$. Therefore it is shown that the operator $A$ generates a $C_{0}$-semigroup of contractions.

\subsection{Exponential Stability}

We prove that the closed-loop system under the Moment-PD control (15) is exponentially stable by using energy multiplier method (Chen et al., 1987; Rao, 1994).

Theorem 2. The closed-loop system (26) is exponentially stable.

PROOF. Let us introduce the following energy function:

$$
E(t)=\langle z, z\rangle_{H} .
$$

Then its time derivative along the solutions of the system yields

$$
\begin{aligned}
\dot{E}(t) & =2\langle A z, z\rangle_{H} \\
& =-C\left\{\frac{\tilde{k}_{1}}{\tilde{k}_{2}}(E I)^{2} u_{x x}^{2}(0)+\frac{\tilde{\mu}}{\tilde{k}_{2}^{2}} v_{x}^{2}(0)\right\} \\
& \leq-C_{1} \zeta^{2},
\end{aligned}
$$

where $C_{1}$ is some positive constant and we have used the relation

$$
\zeta^{2} \leq 2\left\{(E I)^{2} u_{x x}^{2}(0)+\frac{v_{x}^{2}(0)}{\tilde{k}_{2}^{2}}\right\} .
$$

Thus we found that $\dot{E}(t)$ is not negative definite. To prove the exponential stability we introduce a new function:

$$
V(t)=E(t)+\varepsilon \sigma(t),
$$

where the positive constant $\varepsilon$ is to be determined and

$$
\sigma(t)=\rho \int_{0}^{l}(x-l) u_{x} v d x+\rho u_{x}(0) \int_{0}^{l}(l-x) v d x .
$$

Using the Cauchy-Schwartz inequality, Poincaré inequality and the inequality

$$
\begin{aligned}
|a||b| \leq 2|a||b| \leq & \delta a^{2}+\frac{b^{2}}{\delta}, \\
& a, b, \delta \in R, \quad \delta>0,
\end{aligned}
$$


it is easy to verify that

$$
|\sigma(t)| \leq C_{2} E(t)
$$

where $C_{2}$ is some positive constant. Therefore we have the following estimate:

$$
\left(1-\varepsilon C_{2}\right) E(t) \leq V(t) \leq\left(1+\varepsilon C_{2}\right) E(t) .
$$

On the other hand the time derivative of $\sigma(t)$ yields

$$
\begin{aligned}
\dot{\sigma}(t)= & -\frac{\rho}{2} \int_{0}^{l} v^{2} d x-\frac{3}{2} E I \int_{0}^{l} u_{x x}^{2} d x \\
& +\frac{E I}{2} l u_{x x}^{2}(0)+\rho v_{x}(0) \int_{0}^{l}(l-x) v d x .
\end{aligned}
$$

Using the Cauchy-Schwartz inequality and the inequality (33), we obtain the estimate

$$
\rho v_{x}(0) \int_{0}^{l}(l-x) v d x \leq \rho l \delta_{1} v_{x}^{2}(0)+\frac{\rho l^{2}}{\delta_{1}} \int_{0}^{l} v^{2} d x,
$$

where $\delta_{1}$ is some positive constant. Thus $\dot{\sigma}(t)$ can be rewritten as

$$
\begin{aligned}
\dot{\sigma}(t) \leq & -\frac{\rho}{2}\left(1-\frac{2 l^{2}}{\delta_{1}}\right) \int_{0}^{l} v^{2} d x-\frac{3}{2} E I \int_{0}^{l} u_{x x}^{2} d x \\
& +\frac{E I}{2} l u_{x x}^{2}(0)+\delta_{1} \rho l v_{x}^{2}(0),
\end{aligned}
$$

from which we have

$$
\begin{aligned}
\dot{V}(t) \leq & -\frac{\rho}{2} \varepsilon\left(1-\frac{2 l^{2}}{\delta_{1}}\right) \int_{0}^{l} v^{2} d x-\frac{3}{2} \varepsilon E I \int_{0}^{l} u_{x x}^{2} d x \\
& -\left\{C \frac{\tilde{k}_{1}}{\tilde{k}_{2}}(E I)^{2}-\frac{E I}{2} \varepsilon l\right\} u_{x x}^{2}(0) \\
& -\left(\frac{C}{\tilde{k}_{2}^{2}} \tilde{\mu}-\varepsilon \delta_{1} \rho l\right) v_{x}^{2}(0) \\
= & -\frac{\rho}{2} \kappa_{1} \int_{0}^{l} v^{2} d x-\frac{E I}{2} \kappa_{2} \int_{0}^{l} u_{x x}^{2} d x \\
& -\kappa_{3} C(E I)^{2} u_{x x}^{2}(0)-\kappa_{4} C \frac{v_{x}^{2}(0)}{\tilde{k}_{2}^{2}}
\end{aligned}
$$

where $\varepsilon<\min \left\{1 / C_{2}, 2 C \tilde{k}_{1} E I /\left(\tilde{k}_{2} l\right), C \tilde{\mu} /\left(\delta_{1} \rho l \tilde{k}_{2}\right)\right\}$, $\kappa_{1}=\varepsilon\left(1-2 l^{2} / \delta_{1}\right), \kappa_{2}=3 \varepsilon, \kappa_{3}=\tilde{k}_{1} / \tilde{k}_{2}-$ $\varepsilon l /(2 E I C), \kappa_{4}=\tilde{\mu}-\varepsilon \delta_{1} \rho l \tilde{k}_{2}^{2} / C$. Thus we obtain the following estimate from the (32), (34) and (35):

$$
\dot{V}(t) \leq-K E(t) \leq-\frac{K}{1+\varepsilon C_{2}} V(t),
$$

where $K=\min \kappa_{i}(i=1, \ldots, 4)$. Therefore we obtain

$$
E(t) \leq \frac{1+\varepsilon C_{2}}{1-\varepsilon C_{2}} e^{-\frac{K}{1+\varepsilon C_{2}} t} E(0),
$$

which means the exponential stability of the closedloop system under the Moment-PD control,

$$
\|z\|_{H} \leq \sqrt{\frac{1+\varepsilon C_{2}}{1-\varepsilon C_{2}}} e^{-\frac{K}{2\left(1+\varepsilon C_{2}\right)} t}\|z(0)\|_{H} .
$$

Although the exponential stability of the closed-loop system (17), (18), (20) and (21) is established, the exponential stability of the system (2)-(5) and (15) is not shown yet. We need to show that the trajectory of $w, w_{t}, \theta, \dot{\theta}$ and $\lambda$ decays exponentially. Finally we can obtain the following theorem.

Theorem 3. The trajectory of $w, w_{t}, \theta, \dot{\theta}$ and $\lambda$ decays exponentially. That is, there exists positive constants $M_{i}(i=1, \ldots, 5)$ and $\delta$ such that

$$
\begin{aligned}
& \qquad \begin{array}{l}
\left\|w-w^{d}\right\|_{L^{2}} \leq M_{1} e^{-\delta t}, \quad\left\|w_{t}\right\|_{L^{2}} \leq M_{2} e^{-\delta t}, \\
\left\|\theta-\theta^{d}\right\| \leq M_{3} e^{-\delta t}, \quad\|\dot{\theta}\| \leq M_{4} e^{-\delta t}, \\
\left\|\lambda-\lambda^{d}\right\| \leq M_{5} e^{-\delta t},
\end{array} \\
& \text { where }\|\cdot\|_{L^{2}}^{2}=\int_{0}^{l} \cdot{ }^{2} d x \text { and }\|\cdot\|^{2}={ }^{2} .
\end{aligned}
$$

PROOF. Let us denote equation (36) as

$$
\|z\|_{H} \leq M e^{-\delta t} \text {. }
$$

From this equation, we obtain the following estimates:

$$
\|y\|_{L^{2}} \leq K_{0} M e^{-\delta t}, \quad\left\|y_{t}\right\|_{L^{2}} \leq K_{1} M e^{-\delta t},
$$

where we used Poincaré inequality in the derivation of the first term and $K_{0}, K_{1}$ are some positive constant. Further it is easy to see that the estimate

$$
y_{x}^{2}(0, t) \leq K_{2} \int_{0}^{l} y_{x x}^{2} d x
$$

is satisfied by using the Cauchy-Schwartz inequality and (33), where $K_{2}$ is some positive constant. This means

$$
\left\|y_{x}(0, t)\right\| \leq \sqrt{\frac{2 K_{2}}{E I}} M e^{-\delta t} .
$$

For $z(0) \in D(A)$, we can show that

$$
\|z\|_{D(A)} \leq M_{1} e^{-\delta t}\|z(0)\|_{D(A)} \equiv M_{2} e^{-\delta t},
$$

where $M_{1}=\sqrt{\left(1+\varepsilon C_{2}\right) /\left(1-\varepsilon C_{2}\right)}$ and $\|\cdot\|_{D(A)}=$ $\|z\|_{H}+\|A z\|_{H}$. Now the following estimate is obtained as same as the derivation of (38):

$$
y_{x t}^{2}(0, t) \leq K_{3} \int_{0}^{l} y_{t x x}^{2} d x,
$$

where $K_{3}$ is some positive constant. Thus

$$
\frac{E I}{2 K_{3}} y_{x t}^{2}(0, t) \leq\|A z\|_{H}^{2} \leq M_{2}^{2} e^{-2 \delta t},
$$

which means

$$
\left\|y_{x t}(0, t)\right\| \leq \sqrt{\frac{2 K_{3}}{E I}} M_{2} e^{-\delta t}
$$

Next we consider the decay of $E I y_{x x x}(l, t)$. To prove this fact we introduce the following function:

$$
\begin{aligned}
h_{1}= & \frac{C}{2}\left\{-E I y_{x x}(0, t)+\frac{1}{\tilde{k}_{2}} y_{x t}(0, t)\right\}^{2} \\
& +\frac{E I}{2} \int_{0}^{l} y_{t x x}^{2} d x .
\end{aligned}
$$

Then, by using (33) and (41), we obtain

$$
\begin{aligned}
h_{1} \geq & \frac{C}{2}\left(1-\delta_{2}\right)(E I)^{2} y_{x x}^{2}(0, t) \\
& +\frac{C}{2}\left(\frac{1}{\tilde{k}_{2}^{2}}-\frac{1}{\tilde{k}_{2}^{2} \delta_{2}}+\frac{E I}{K_{3} C}\right) y_{x t}^{2}(0, t),
\end{aligned}
$$


where $\delta_{2}$ satisfies $\delta_{2} \in\left(1 /\left(1+\tilde{k}_{2}^{2} E I /\left(K_{3} C\right)\right), 1\right)$. Thus, using this estimate, we found that

$$
\|z\|_{D(A)}^{2} \geq K_{4}\left\{\int_{0}^{l} y_{x x x x}^{2} d x+(E I)^{2} y_{x x}^{2}(0, t)\right\},
$$

for $K_{4} \leq \min \left\{C\left(1-\delta_{2}\right) / 2,(E I)^{2} /(2 \rho)\right\}$. On the other hand we can show that there exists some positive constant $K_{5}$ such that

$$
\begin{aligned}
& (E I)^{2} y_{x x x}^{2}(l, t) \\
& \quad \leq K_{5}\left\{\int_{0}^{l} y_{x x x x}^{2} d x+(E I)^{2} y_{x x}^{2}(0, t)\right\},
\end{aligned}
$$

by using the Cauchy-Schwartz inequality, Poincaré inequality and (33). From (43) and (44) we can obtain the estimate about the decay of $E I y_{x x x}(l, t)$ :

$$
\left\|E I y_{x x x}(l, t)\right\| \leq \sqrt{\frac{K_{5}}{K_{4}}} M_{2} e^{-\delta t} .
$$

Therefore we have the desired result by using (6), (16), (37), (39), (42), (45), and by considering the relation $\lambda(t)=E I y_{x x x}(l, t)$.

\section{CONCLUSIONS AND FUTURE WORKS}

In this paper, we consider force control problem for constrained one-link flexible arm. We proposed Moment-PD control which has a feedback of the bending moment at the root of the flexible arm and its time derivative. Hence this controller is very simple in its structure. Furthermore this controller does not need information about the contact force and the rotational angle. Then exponential stability of the closed-loop system was proven by using energy multiplier method.

The future work is the experimental validation of the proposed Moment-PD control.

\section{REFERENCES}

Balas, M.J. (1978). Active control of flexible systems. J. Opt. Theory and Appl. 25, 415-436.

Chen, G., M.C. Delfour, A.M. Krall and G. Payre (1987). Modeling, stabilization and control of serially connected beams. SIAM J. Control Optim. 25(3), 526-546.

Chiou, B.C. and M. Shahinpoor (1988). Dynamic stability analysis of a one-link force-controlled flexible manipulator. J. Robot. Syst. 5(5), 443-451.

Conrad, F. and Ö. Morgül (1998). On the stabilization of a flexible beam with a tip mass. SIAM J. Control Optim. 36(6), 1962-1986.

Luo, Z. (1993). Direct strain feedback control of flexible robot arms : New theoretical and experimental results. IEEE Trans. Automat. Contr. 38, 1610-1622.
Luo, Z.H. and B.Z. Guo (1997). Shear force feedback control of a single link flexible robot with revolute joint. IEEE Trans. Automat. Contr. 42, 5365.

Luo, Z.H. and D.X. Feng (1999). Nonlinear torque control of a single-link flexible robot. J. Robot. Syst. 16, 25-35.

Matsuno, F. and S. Kasai (1998). Modeling and robust force control of constrained one-link flexible arms. J. Robot. Syst. 15(8), 447-464.

Morgül, Ö. (1991). Orientation and stabilization of a flexible beam attached to a rigid body : Planar motion. IEEE Trans. Automat. Contr. 36, 953962.

Morita, Y., F. Matsuno, Y. Kobayashi, M. Ikeda, H. Ukai and H. Kando (2002). Lyapunov-based force control of a flexible arm considering bending and torsional deformation. In: Proc. IFAC World Cogress. Session slot T-Tu-M04, No. 1 (CD-ROM).

Morita, Y., Y. Kobayashi, H. Kando, F. Matsuno, T. Kanzawa and H. Ukai (2001). Robust force control of a flexible arm with a nonsymmetric rigid tip body. J. Robot. Syst. 18(5), 221-235.

Pazy, A. (1983). Semigroups of Linear Operators and Applications to Partial Differential Equations. Springer-Verlag. New York.

Rao, B. (1994). Recent progress in non-uniform and uniform stabilization of the scole model with boundary feedbacks. In: Boundary control and variation (J.P. Zolésio, Ed.). pp. 357-365. Marcel Dekker, Inc.

Siciliano, B. and L. Villani (2000). Parallel force and position control of flexible manipulators. IEE Proc. Control Theory Appl. 147(6), 605-612. 